\title{
Thermal Analysis of the MC-1 Chamber/Nozzle
}

\author{
Darrell Davis \\ NASA/Marshall Space Flight Center, ED25, MSFC, AL \\ (256) 544-7257, darrell.davis@msfc.nasa.gov
}

\begin{abstract}
This paper will describe the thermal analysis techniques used to predict temperatures in the film-cooled ablative rocket nozzle used on the MC-1 $60 \mathrm{~K}$ rocket engine. A model was developed that predicts char and pyrolysis depths, liner thermal gradients, and temperatures of the bondline between the overwrap and liner. Correlation of the model was accomplished by thermal analog tests performed at Southern Research, and specially instrumented hot fire tests at the Marshall Space Flight Center. Infrared thermography was instrumental in defining nozzle hot wall surface temperatures. In-depth and outboard thermocouple data was used to correlate the kinetic decomposition routine used to predict char and pyrolysis depths. These depths were anchored with measured char and pyrolysis depths from crosssectioned hot-fire nozzles. For the X-34 flight analysis, the model includes the ablative Thermal Protection System (TPS) material that protects the overwrap from the recirculating plume. Results from model correlation, hot-fire testing, and flight predictions will be discussed
\end{abstract}

\section{INTRODUCTION}

The MC-1 (previously known as Fastrac) program provides a low-cost, $60,000-1 \mathrm{~b}(60 \mathrm{~K})$ thrust rocket engine to the aerospace community. Part of this low-cost design is an ablative chamber/nozzle assembly that is actively filmcooled with RP1. The chamber/nozzle is designed for one time use only and will be replaced after every flight. The baseline chamber/nozzle consists of a tape-wrapped silica phenolic liner with a filament-wound carbon epoxy overwrap added for extra strength. A filament wound glass phenolic overwrap is also being tested as part of a parallel verification effort. The flight nozzles will have a 30:1 area ratio, however most of the nozzles that have been ground tested have a $15: 1$ ratio.

Concerns during the design phase included: effects of the film cooling, degree of surface recession and the ability to maintain the liner-to-overwrap bondline below $422 \mathrm{~K}$. The insulative properties of the silica phenolic protect the bondline during firing (typically 150 seconds), however the "soakback" effect causes the bondline to exceed the limit after shutdown and to potentially create a debond. The soakback effect was mentioned as a design problem at the Preliminary Design Review. To address this, as well as the other desing concerns, extra tests were added to gather data to help refine the thermal model. As the design progressed, it was obvious that the liner had to be thicker to protect the bondline. This was an undesirable solution since it increased the weight of the nozzle. The solution was to thicken the liner as much as possible only at the attach rings and add to shear pins to distribute the load into the nozzle in case the rings still came loose. A representation of the final design is shown in Figure 1.

\section{MODEL DESCRIPTION}

A one-dimensional SINDA model was developed to predict in-depth temperature response and bondline temperatures for ground tests and for flight conditions. The SINDA model consisted of 45 finite element nodes across the thickness of the silica phenolic liner and 5 nodes through the graphite epoxy overwrap. A non-linear grid 
was used to capture the high gradients near the surface while minimizing the overall number of nodes. Material properties for the virgin and charred silica phenolic were obtained through testing at Southern Research, Inc. (SORI) in Birmingham, AL. Preliminary hot gas temperature predictions were provided by the CFD group at the Marshall Space Flight Center. SINDAVCMA, a kinetic decomposition routine, based on the Arrhenius equation, was added to account for the effect of material decomposition and pyrolysis gas formation. Cork was added to the external nozzle surfaces to protect the graphite epoxy from the plume recirculation environments during flight. $A B L$, an in-house developed code that can be coupled with SINDA, was used to size the cork. ABL, described by Clayton (1996), uses an empirically derived recession rate versus heat rate curve to calculate surface recession of the cork while tracking thermal capacitance and conduction path lengths to calculate heat transfer through the receding material. This is the first model generated at MSFC that has incorporated SINDAVCMA and ABL to account for material decomposition of two different materials experiencing two different environments.

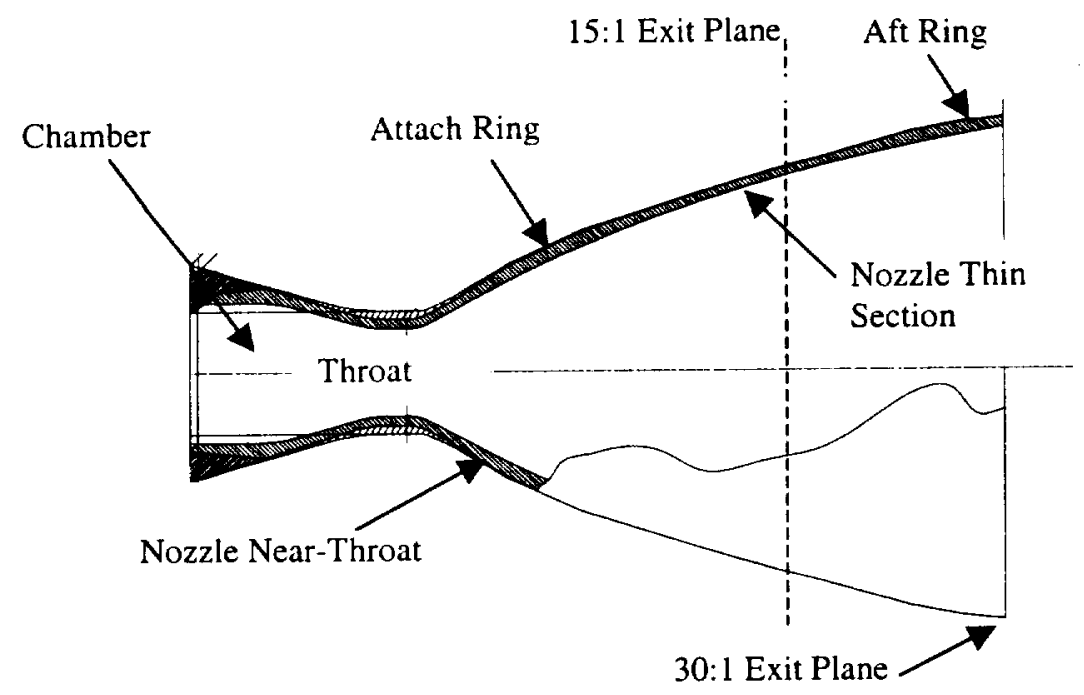

FIGURE 1. MC-1 Chamber/Nozzle Baseline Configuration.

\section{MODEL DESCRIPTION}

A one-dimensional SINDA model was developed to predict in-depth temperature response and bondline temperatures for ground tests and for flight conditions. The SINDA model consisted of 45 finite element nodes across the thickness of the silica phenolic liner and 5 nodes through the graphite epoxy overwrap. A non-linear grid was used to capture the high gradients near the surface while minimizing the overall number of nodes. Material properties for the virgin and charred silica phenolic were obtained through testing at Southern Research, Inc. (SORI) in Birmingham, AL. Preliminary hot gas temperature predictions were provided by the CFD group at the Marshall Space Flight Center. SINDA/CMA, a kinetic decomposition routine, based on the Arrhenius equation, was added to account for the effect of material decomposition and pyrolysis gas formation. Cork was added to the external nozzle surfaces to protect the graphite epoxy from the plume recirculation environments during flight. $A B L$, an in-house developed code that can be coupled with SINDA, was used to size the cork. ABL uses an empirically derived recession rate versus heat rate curve to calculate surface recession of the cork while tracking thermal capacitance and conduction path lengths to calculate heat transfer through the receding material. This is the first model generated at MSFC that has incorporated SINDA/CMA and $\mathrm{ABL}$ to account for material decomposition of two different materials experiencing two different environments. 


\section{TESTING}

The first data used to correlate the model came from Thermal Analog tests performed at SORI. The test provided one-dimensional heating of a $50.8 \mathrm{~mm} \times 50.8 \mathrm{~mm} \times 21.6 \mathrm{~mm}$ coupon of silica phenolic/graphite epoxy by exposing the coupon surface to a resistively heated graphite heater. The coupon surface was heated at rates that simulated actual engine firings. A total of three samples were tested. The first two tests runs were for 150 seconds and the third was for 230 seconds. Temperature of the surface, backside, and five in-depth locations were recorded with thermocouples. Depths of the imbedded thermocouples were determined by CT techniques. The recorded surface temperature was used as an input to the SINDA model and temperatures were predicted at the measured thermocouple depths. Because of uncertainty in the char properties, and the unknown material properties in the pyrolysis region, material properties were adjusted until the SINDA model results matched the results from the Thermal Analog Tests. Figure 2 shows typical correlation results. Char and heat-affected depths were also taken from these samples. This data was used to anchor the kinetic decomposition routine.

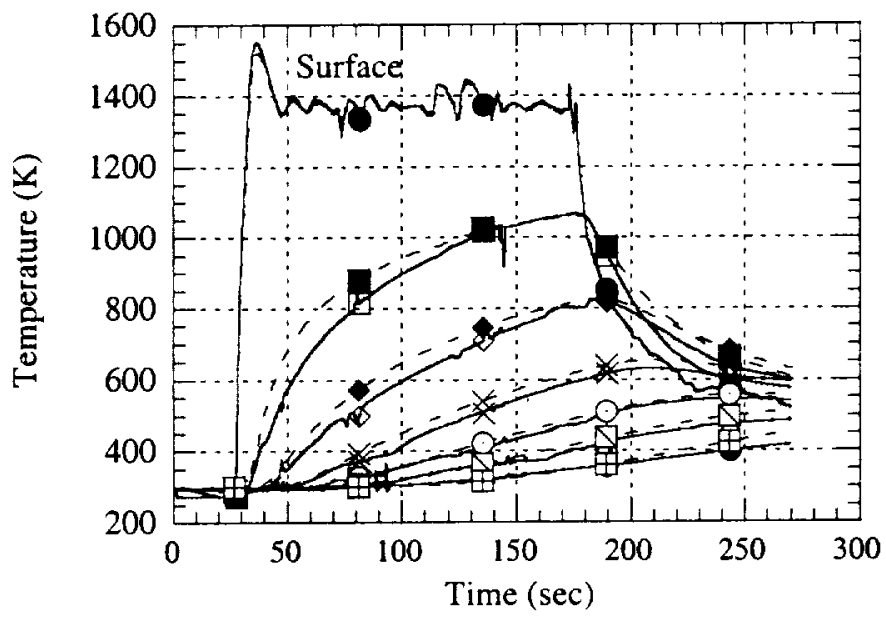

FIGURE 2. Model Comparison to SORI Thermal Analog Tests. Solid lines represent test results. Dotted lines represent model results.

A significant amount of data was gathered from hot fire component testing performed at MSFC's Test Stand 116. These were static tests to verity the design of the Thrust Chamber Assembly (TCA). Propellants were pressure-fed instead of using actual MC-1 turbomachinery. During this series of tests, an attempt was made to gather as much data as resources would allow. This data would prove valuable to the validation of the thermal math model. All static tests had two thermocouples installed in holes drilled through the aft-facing surface of the silica phenolic. One was a bare Type $C$ thermocouple mounted flush with the liner surface to measure surface temperature, the other was a shielded Type C thermocouple mounted with the bead about 0.125 " into the flow to measure the local hot gas temperature. An infrared scanner was also used to determine the interior surface temperature. The results from these two methods matched well and indicated that the actual gas temperature was lower than predicted.

In two of the early tests, designated $60 \mathrm{~K} \# 1$ and $60 \mathrm{~K} \# 2$, thermocouple plugs were used to measure in-depth temperature response. This process was developed during earlier technology development programs at MSFC. The plugs were $6.35 \mathrm{~mm}$ silica phenolic cylinders into which were imbedded three thermocouples. The plugs were laid up in the same manner as the liner material. Care had to be taken during installation to ensure proper placement of the thermocouple leads within the plug. A hole was drilled into the silica phenolic liner from the backside to within a nominal $2.54 \mathrm{~mm}$ from the surface and the plugs were installed into these holes. Plugs were placed at four axial locations and two radial locations for each axial location. Depths of these thermocouples were again obtained by using CT techniques. A sample thermocouple measurement versus model prediction is presented in Figure 3. 


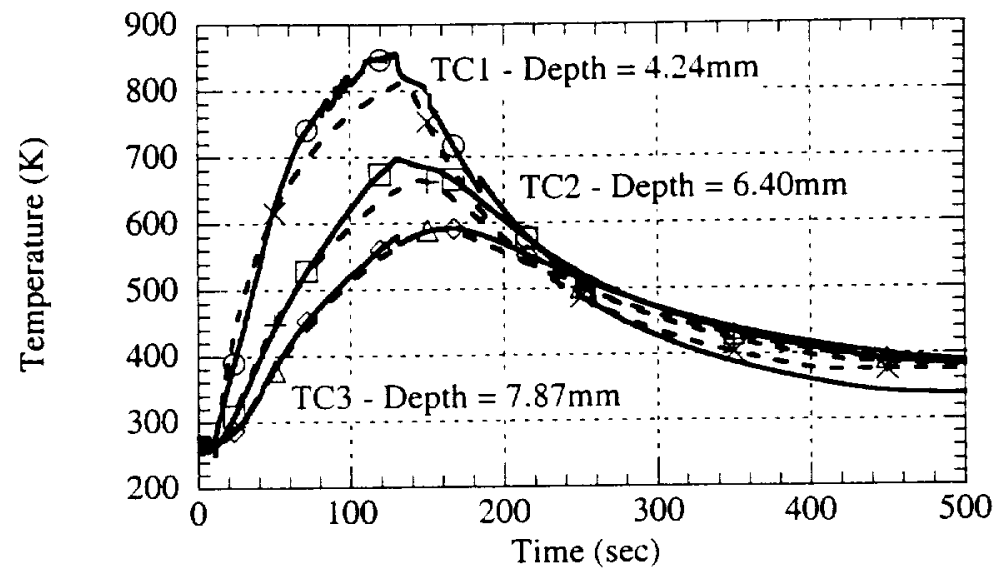

FIGURE 3. Model versus Test Data for Test $60 \mathrm{~K} \# 2$, Plug $1 \mathrm{~A}$. Solid lines represent test results.

Dotted lines represent model preditions.

After testing, the $60 \mathrm{~K} \# 1$ and $60 \mathrm{~K} \# 2$ nozzles were sliced axially and post-test char and heat-affected depths were measured. This data was used to verify the decomposition routine. Data from these tests also confirmed that there was no surface recession of the silica phenolic even during a 130 -second test. Since multiple short-duration tests were to be performed on each nozzle, the model was relied upon to verify that the bondline had not exceeded its temperature limit, and could be tested again. Therefore all static tests also had exterior thermocouples placed at key axial locations. These thermocouples provided data to anchor the model for each test and ensured reliable bondline predictions.

The next series of tests were performed at the Stennis Space Center (SSC) in Pascagoula, Mississippi. These tests were system level validation tests that incorporated the turbomachinery with the TCA. Since drilling was not allowed on these nozzles, and the test stand does not provide adequate placement for the infrared scanner, external thermocouples were the only source of data on the tests at SSC. The H2 series tests were performed on a 15:1 nozzle with a glass phenolic overwrap. Results from thermal analysis and test data showed that the glass phenolic and graphite epoxy overwraps would perform very similarly thermally. Test $\mathrm{H} 2 \mathrm{~A}-2 \mathrm{~B}$ and Test H2B-2 both ran for the planned full duration of 24 and 155 second, respectively. Again, the model predictions agreed with the thermocouple data, providing more validity to the model.

These nozzles were also instrumented with strain gauges to allow structural analysts to verify their models. Data from these strain gauges can show where a debond has occurred. Figure 4 shows strain gauge and thermocouple data plotted together at the exit plane, where a visual examination confirmed a debond. The spike in the strain gauge data indicates a debond occurred at that time. At the same time, the thermocouple data changes slope. This is also an indication of a debond. When the graphite epoxy overwrap debonds from the liner, the conduction path from the liner to overwrap is broken. The overwrap is then more heavily influenced by convective cooling from the ambient temperature than by the radiant heating now produced by the liner. This causes a change in slope in the thermocouple response.

The nozzle near-throat region is an area of concern for potential debonding. In this section, the overwrap is becoming thinner due to an increase in local area ratio and the liner is not as thick as it is further downstream at the attach ring. Visual observations cannot reveal a debond in this region, and on-pad non-destructive evaluation (NDE) methods have not been developed to a point where they are feasible to use in small, tight spaces. Strain gauge data from the near-throat region of Test H2B-2 seems to indicate a debond. When the thermocouple data is plotted alongside the strain gauge data it may also show indications of a debond by the change of slope in the temperature trace. 


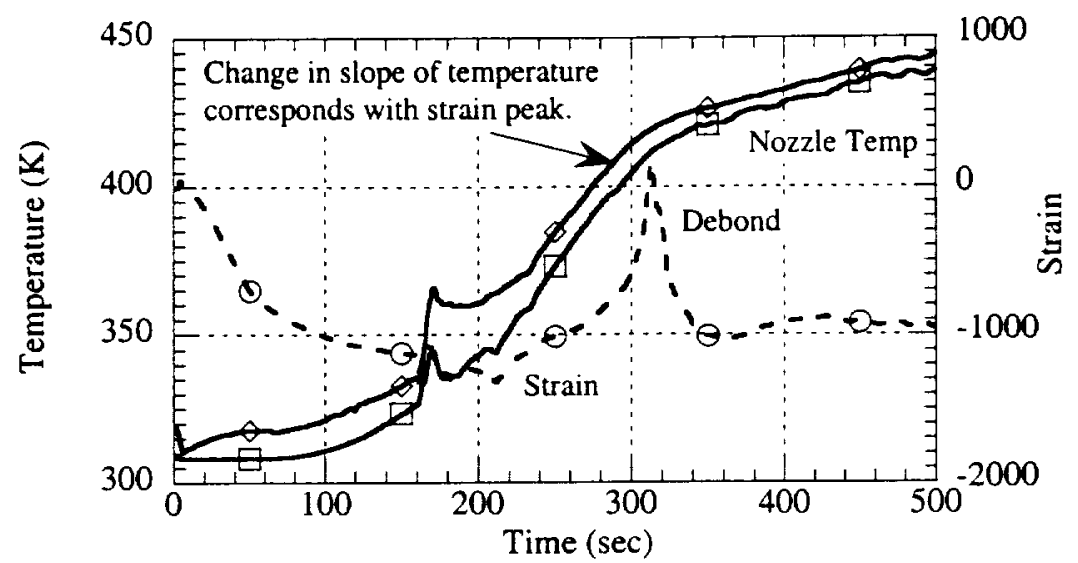

FIGURE 4. Thermocouple and Strain Gauge Data From Test H2B-2 at the Exit Plane.

The nozzle near-throat region is an area of concern for potential debonding. In this section, the overwrap is becoming thinner due to an increase in local area ratio and the liner is not as thick as it is further downstream at the attach ring. Visual observations cannot reveal a debond in this region, and on-pad non-destructive evaluation (NDE) methods have not been developed to a point where they are feasible to use in small, tight spaces. Strain gauge data from the near-throat region of Test H2B-2 seems to indicate a debond. When the thermocouple data is plotted alongside the strain gauge data it may also show indications of a debond by the change of slope in the temperature trace.

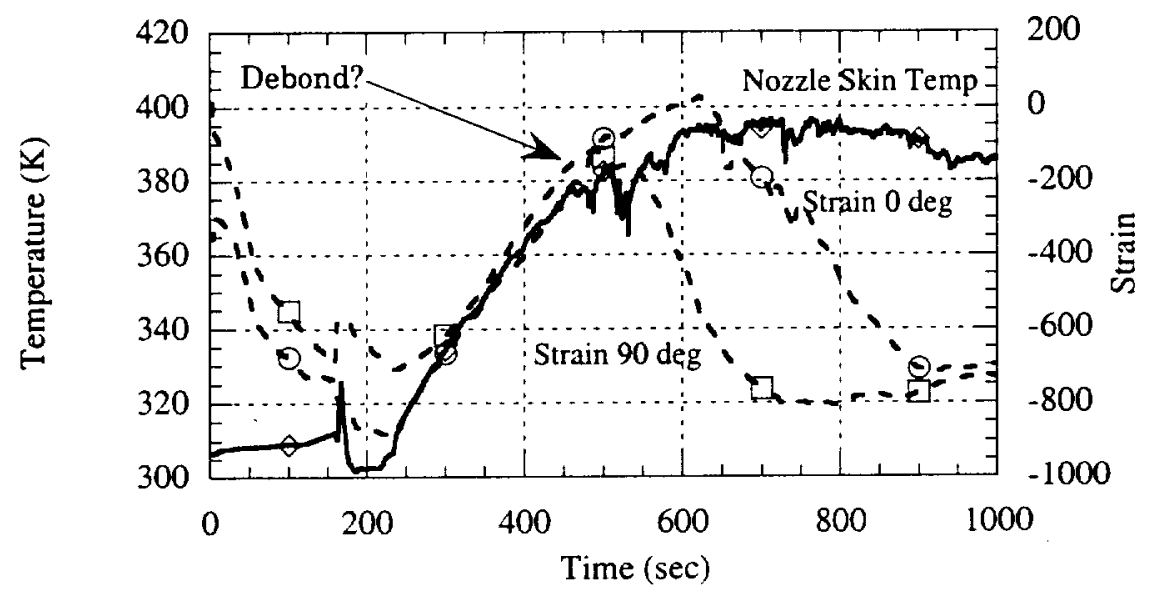

FIGURE 5. Thermocouple and Strain Gauge Data From Test H2B-2 Near Throat.

Post test laboratory NDE could not positively declare a debond at this location, but did reveal a low-density area. It is possible that this configuration of thermocouples and strain gauges could be used for health monitoring of the nozzle during ground tests.

\section{FLIGHT PREDICTIONS}

During flight, the overwrap forward of the heat shield will be exposed to environments generated inside the aft compartment. The nozzle itself will contribute to this environment, especially post-firing. Flight exterior nozzle temperatures were provided to Orbital Sciences Corporation for inclusion to their aft compartment model. Aft of the heat shield, the nozzle will be exposed to recirculation of the plume. MSFC's CFD group provided plume 
recirculation environments. $A B L$, an in-house code that runs concurrently with SINDA, was used to size the thermal protection system (TPS) materials. Two materials were selected for analysis: cork, and Marshall Convergent Coating (MCC-1). MCC-1 is a sprayable ablator developed at MSFC containing cork, glass ecospheres and an epxoy resin. It is currently used as the main acreage TPS on the Solid Rocket Boosters. Results from the analysis showed that $6.35 \mathrm{~mm}$ of either material would protect the graphite epoxy overwrap to $422 \mathrm{~K}$. Since MCC-1 is sprayable, it requires the programming of a computer to follow the specific geometry of the nozzle. Since this made the MCC-1 more expensive for a short production run, the program chose to use cork as the external TPS material. RT-455, will be used as a closeout material and the entire TPS system will be covered with Acrymax paint.

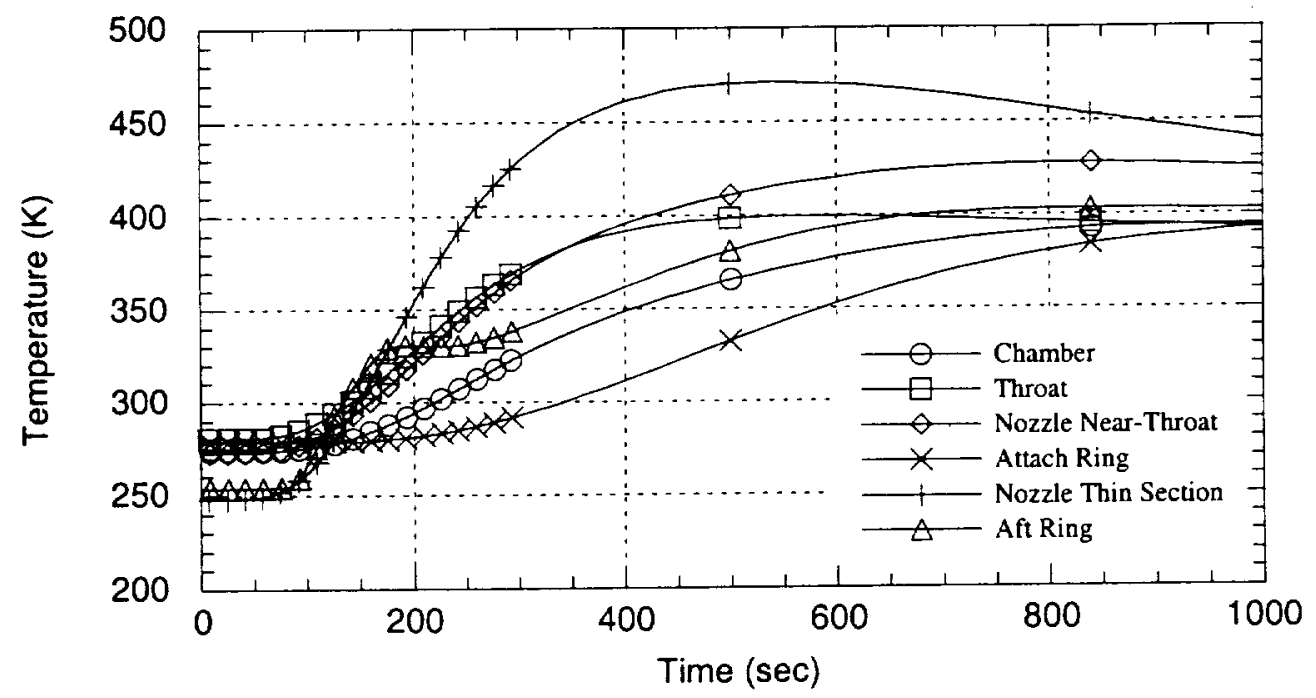

FIGURE 6. Bondline Flight Predictions.

Once the model had been proven reliable and able to match test results, it was used to provide two-dimensional thermal distributions to structural analysts. The kinetic decomposition routine could not be adapted to a twodimensional grid. Since there was very little difference in local plume temperatures and therefore no $\Delta T$ to drive axial conduction, it was determined that an interpolation of 1-D results would provide the necessary data. The analytical nozzle was separated into 281 -D slices. Each slice was run with its corresponding gas temperature, heat transfer coefficient, silica phenolic thickness, and graphite epoxy thickness to provide a thermal gradient profile at that location. The results were interpolated onto a 2-D PATRAN finite element mesh. Results provided for structural analysis included hot and cold extremes for both ground and flight.

\section{CONCLUSIONS}

Because of an extensive test program that generated data used to correlate the model, the model can be trusted to give reliable results. These results indicate that during a 150 -second engine burn, all bondlines will remain below the $338 \mathrm{~K}$ bondline limit. However, this limit will be violated during the soakback and will potentially cause a debond. These results were forwarded to the project office and structural changes, such as shear pins, are currently being investigated.

\section{REFERENCES}

Clayton, J.L., "SINDA/ABL Solution Routine Updates," NASA/MSFC Memo ED63(25-96), July 1996. 